Open Access

\title{
The importance of intrinsically photosensitive retinal ganglion cells and implications for lighting design
}

\author{
Dingcai Cao ${ }^{1^{*}}$ (1) and Pablo A. Barrionuevo ${ }^{1,2}$
}

\footnotetext{
* Correspondence: dcao98@uic.edu ${ }^{1}$ Visual Perception Laboratory,

Department of Ophthalmology and Visual Sciences, University of Illinois at Chicago, Chicago, IL 60612, USA Full list of author information is available at the end of the article
}

\begin{abstract}
We reviewed the role of melanopsin-containing intrinsically photosensitive retinal ganglion cells (ipRGCs) in light-dependent functions, including circadian rhythm that is important for health and visual perception. We then discussed the implications for lighting design.
\end{abstract}

Keywords: Melanopsin, ipRGC, Photoreceptors, Circadian, Visual perception, Color,

Contrast Sensitivity, Health, Lighting, LED, Lighting Design

\section{Introduction}

In addition to rod and cone photoreceptors, there exists a third class of photoreceptors in the mammalian retina, called intrinsically photosensitive retinal ganglion cells (ipRGCs). IpRGCs were first discovered in mice in $2002[1,2]$ and then in primates and human in 2005 [3]. IpRGCs express melanopsin, a photopigment with a peak sensitivity at $\sim 482 \mathrm{~nm}$ (see Fig. 1 for human photoreceptor spectral sensitivity functions [4-7]. In addition to intrinsic melanopsin-mediated photoresponses, ipRGCs also receive synaptic inputs from rods and cones. The combination of melanopsin activation, rod and cone inputs enable ipRGCs to signal a large dynamic range of light levels in the environment (by a factor of 10 billion from dim starlight to bright sunlight) [8].

IpRGCs project to brain areas such as the suprachiasmatic nucleus $(\mathrm{SCN})$ to mediate circadian photoentrainment [9] or the olivary pretectal nucleus (OPN) to control pupil light responses [10]. IpRGCs also provide light information to the pineal melatonin production system $[11,12]$ and sleep regulation system [13, 14], and modulate cognitive function [15], alertness (e. g. [16, 17]), body temperature (e. g. [18, 19]), mood and emotion [20]. Therefore, ipRGCs are considered to be the primary photoreceptors for sub-conscious non-image-forming (NIF) functions that are important for our normal biological activities and health. IpRGCs are also found to project to the lateral geniculate nucleus (LGN), the thalamic relay to the visual cortex, and therefore the melanopsin-based signal may also contribute to conscious image-forming (IF) vision [3]. Here we reviewed the importance of melanopsin activation on health, pupil responses and visual perception and the implications for lighting design. 


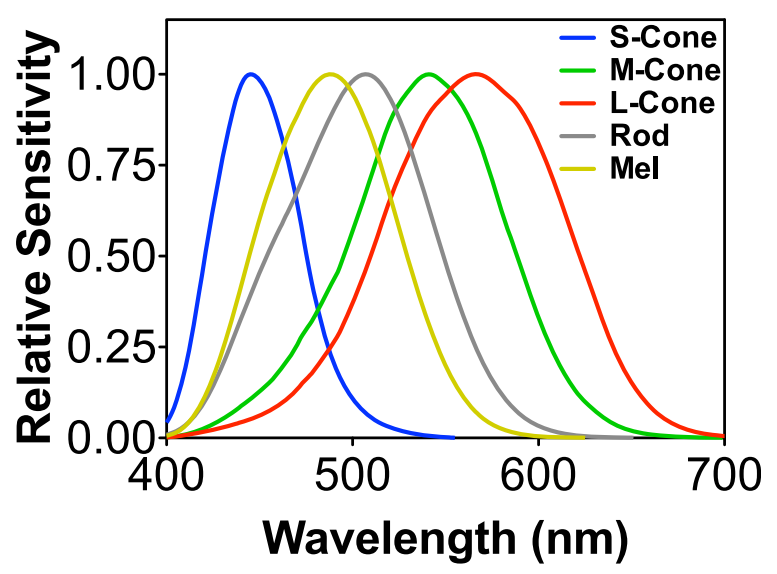

Fig. 1 Human photoreceptor spectral sensitivity functions

\section{Review}

\section{Impact on health}

A normal ipRGC function is important for normal biological, physiological activities and health. IpRGCs are found to be important for several non-retinal diseases, such as sleep disorders, seasonal affective disorder, mood disorders, and migraines [20]. One of the critical mechanisms for ipRGCs affect health is their photic input to the circadian system.

In a simple configuration, the central circadian system can be conceptualized as having three components: (1) the central clock, which generates the rhythms, (2) input pathways that provide signals to synchronize the central clock, and (3) output pathways that convey the central clock signal to other regulatory systems in the brain and body (Fig. 2). The central circadian clock exists in the SCN, a tiny region located in the hypothalamus, sitting right above the optic chiasm. There are three major neural input pathways to the SCN: (1) retinal photoreceptors that transmit the light signal to the SCN via the retinohypothalamic tract, (2) neuronal projections from the raphe nuclei,

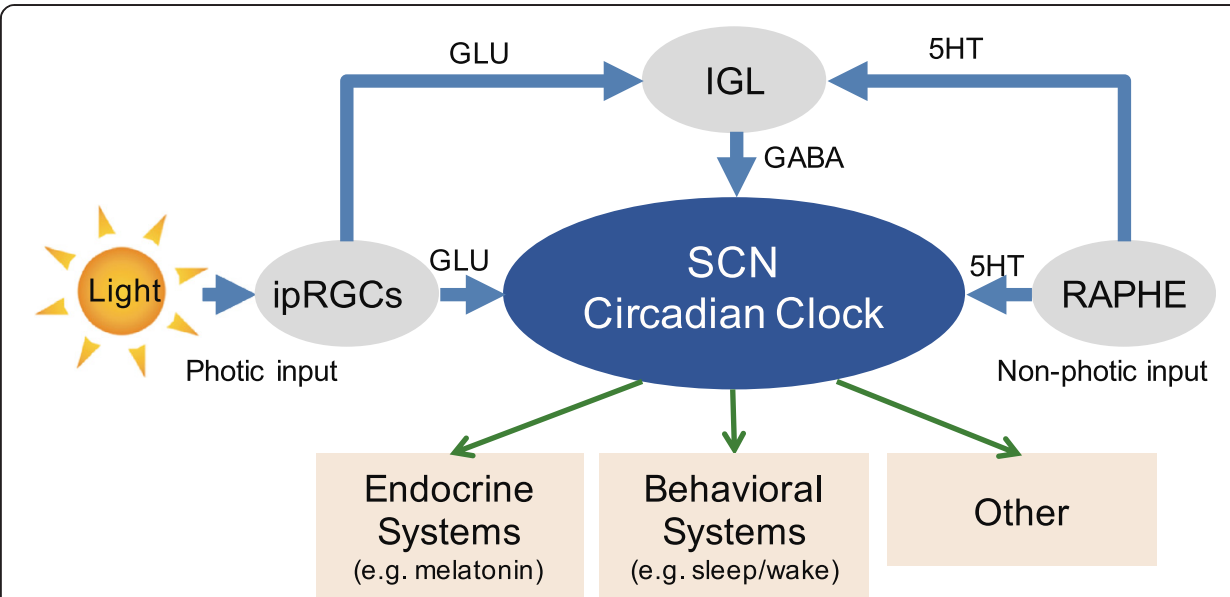

Fig. 2 The three main components of the central circadian system: 1) central clock in the SCN; 2) input pathways, and 3) output pathways. IpRGCs provide photic inputs and RAPHE provides non-photic inputs to the SCN. IGL, which receives inputs from both ipRGCs and RAPHE, also send signals to the SCN. The neurotransmitter in each pathway is shown: GLU for glutamate; GABA for gamma-Aminobutyric acid; 5HT (serotonin). RAPHE: the raphe nuclei; IGL: the intergeniculate leaflet 
which provide non-photic inputs, and (3) neuronal projections from the intergeniculate leaflet (IGL), which also receives inputs from the retina and raphe nuclei. The output pathways are implicated in the control of the endocrine system (such as melatonin release), and other brain and body regions controlling various behaviors such as sleep/ wake [21, 22].

The correct timing of the central circadian clock relative to the environment is essential for optimal sleep, waking functions and health. In humans, the central circadian clock has an average endogenous period slightly greater than $24 \mathrm{~h}(\sim 24.2 \mathrm{~h})$ [23]. Daily input signals are required to shift the clock earlier (phase advance) to synchronize the clock's timing to the external 24-h solar day, and light is the strongest zeitgeber ("time giver") to the central circadian clock. In humans, light in the evening or first part of the night causes the clock to shift rhythms later (phase delay) and light in the morning shift the clock earlier (phase advance). Thus morning light is essential for producing corrective daily phase advances in humans, while evening light can produce phase delays, which exacerbate the human clock's endogenous tendency to drift later and promote circadian misalignment. Circadian misalignment can lead to difficulty in falling asleep, maintaining sleep, excessive daytime sleepiness lower quality of life, worsen mood and well-being, worsen depression, reduce cognitive performance and increase rates of myocardial infarction and cancer [20, 21, 24-28].

IpRGCs can not only influence health through their photic input to the circadian system, but also provide direct information to brain areas that are important for sleep, cognition, and mood [35]. In addition, ipRGCs are found to be related to retinal diseases such as glaucoma and age-related macular degeneration [29]. Thus, ipRGCs exerts a major influence on circadian timing, which in turn impacts mental and physical health.

\section{Impact on pupil responses}

IpRGCs send photic signals to the OPN to control pupil light responses [10]. Pupil size variation produces a number of changes in retinal stimulation to affect visual functions, including retinal illuminance (the amount of light falling into the retina), the ratio of $\mathrm{rod} /$ cone stimulation, spectral sensitivity and spatial resolution [30]. In fact, the melanopsin spectral sensitivity function estimated from in vivo post-illumination pupil response (PIPR) in humans or macaques [31] is almost identical to that measured from in vitro ipRGC recording in macaques (peak at $482 \mathrm{~nm}$ ) [3]. Therefore, pupil light reflex measurement can be used as a functional marker of ipRGC response. We now know that tonic pupil responses are driven preferentially by melanopsin activation, while rod and cones are combined to signal phasic pupil responses [5, 32]. Further, compared with cone-mediated PIPR, the melanopsin-mediated PIPR has long integration duration [33] and large spatial summation area [34].

\section{Impact on visual perception}

Compared with rod or cone photopigment, melanopsin phototransduction is extremely sluggish [35]. In addition, ipRGCs are rare ( 3,000, only $\sim 0.2 \%$ of total number of RGCs in the primate or human retina) with large cell bodies, dendrite trees and large receptive fields (least 5-10 times more extensive than those for classical RGCs) [3]. It is proposed that ipRGCs sacrifice spatiotemporal resolution to reliably signal ambient 
illumination levels [35]. However, emerging evidences have shown that melanopsin activation in ipRGCs contributes to visual perception directly or indirectly.

IpRGCs act as a photon counter in the same way than a light meter in a camera [3,36]. This unique capability, not shared by other photoreceptors, could serve as a reference for the visual system to optimize light adaptation. Indeed, melanopsin has been found to regulate cone electroretinograms (ERGs) in mice [37] or humans [38]. More recently, it is reported that melanopsin activation level can modulate the spatial/temporal tuning patterns of visual network [36].

Melanopsin activation can affect visual perception directly. It has been reported that humans lacking an outer retina [39] or animals with rods and cones ablated genetically [40] can preserve some light detection functions. In people with normal retinas, melanopsin activation could contribute to brightness discrimination [40], chromatic discrimination [41], color perception [42, 43] and contrast sensitivity [4, 44]. However, the mechanisms for melanopsin activation affecting conscious visual perception are not well-understood.

Visual perception in primates and humans is mediated by three primary visual pathways that transfer visual information from the retina to different layers of the LGN and then subsequently to the visual cortex, including the magnocellular (MC-), parvocellular (PC-), and koniocellular (KC-) pathways $[45,46]$. These pathways combine differential long (L-), middle (M-) and short (S-) wavelength sensitive cone signals. The MC-pathway processes summed L- and M-cone excitations to signal luminance information. The PC-pathway uses the difference in L- and M-cone excitations to mediate the "red-green" chromatic signal. The KC-pathway processes the responses of S-cones opposed to the sum of L- and M-cones to signal the "blue-yellow" chromatic information. However, we have no direct knowledge about how signals arising from melanopsin contribute to the three primary visual pathways to alter visual perception. Using principal component analyses based on the excitations of the melanopsin, rods, S-, M- and L-cones for 9 hyperspectral natural images under 21 natural illuminants, we analyzed the contribution of melanopsin activation to the three primary visual pathways, namely the MC-, PC- and KC- pathways. With only cone excitations considered, the principal components revealed were consistent with the patterns of cone combinations in the MC-, PC- and KC-pathways [47]. Further analysis indicated that melanopsin contributed strongly to the MC- and KC-pathways and weakly to the PC-pathway [5]. It is known that red-green color vision mediated by the PC pathways was evolved much later than the $\mathrm{MC}$ - and KC-pathways [48], therefore from an evolution perspective, it makes senses that melanopsin activation has a weaker input to the PC-pathway.

\section{Implications in lighting design}

Traditionally, lighting industry guidelines followed several scientific principles for efficacy (energy efficiency), light quantity (illumination levels), light quality (color temperature, color rendition, glare, etc.) and lighting uses (ambient, task or accent lighting). The discovery of ipRGCs introduces a new dimension of considerations for lighting or display designs: that is, how to minimize the adverse effect of artificial lights, via ipRGC phototransduction, on mental and physical health while maximize visual functions and energy efficiency (Fig. 3). 


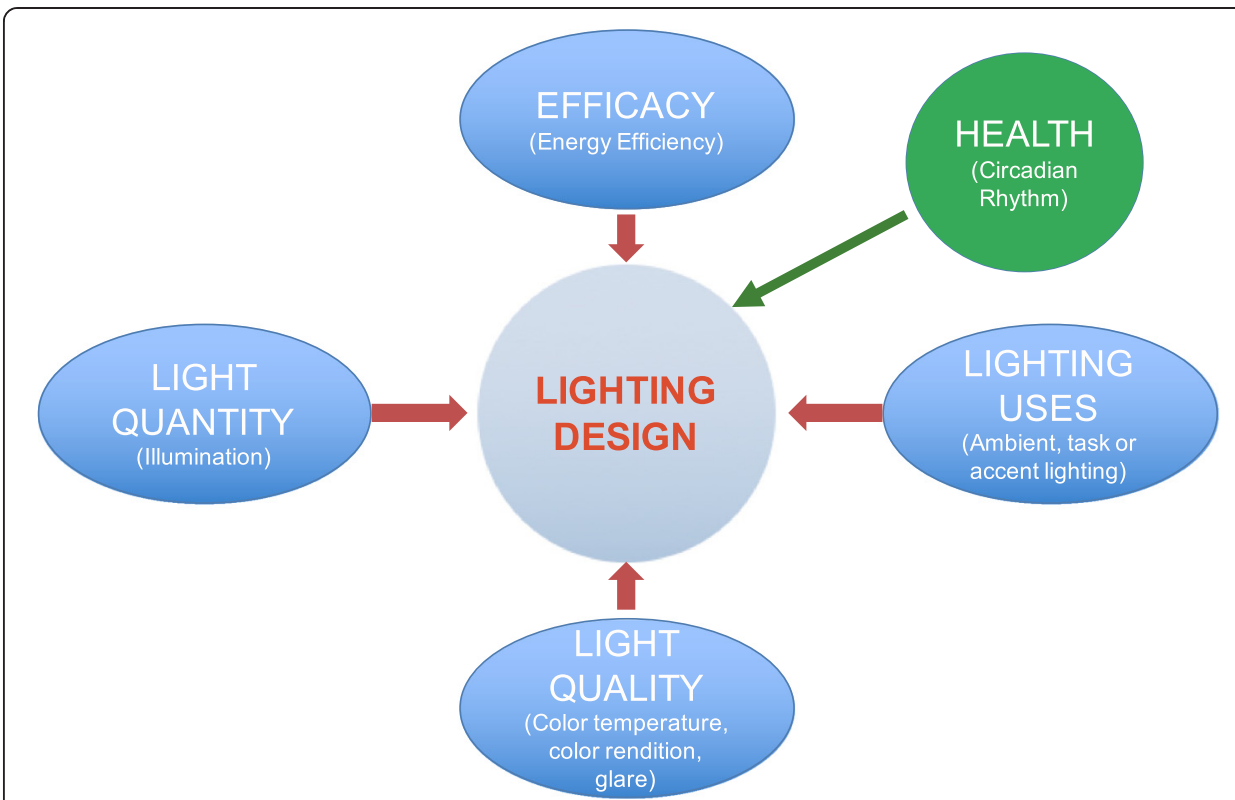

Fig. 3 Scientific principles used for lighting design, including efficacy, light quantity, light quality, lighting uses and health. As the LED light is highly efficient in energy, finding a balance between health and visual performance is a key consideration in future lighting design

Biological adaptation to the sun has evolved over millions of years, however, people in modern society spend a large portion of their time in environments illuminated by artificial lights, working in front of computer displays, watching TV, or interacting with smartphones/tablets for reading, internet surfing, social networking, or video gaming etc. Compared with natural sunlight, the artificial illuminants or display lights are substantially dimmer than daylight, and have different spectral compositions (thus different melanopsin activation levels). Our computation indicated that the artificial illuminants (5 LEDs, 5 High Pressure Sodium lamps, and 27 fluorescents) have significantly lower melanopsin activation level than 25 CIE D natural daylights ([7], Fig. 4). Therefore, indoor workers would experience substantially lower melanopsin excitations compared with outdoor daylight. On the other hand, the artificial lights can be turned on at any time, such as nighttime thus replacing the natural light-dark transition. These abrupt state-light changes will

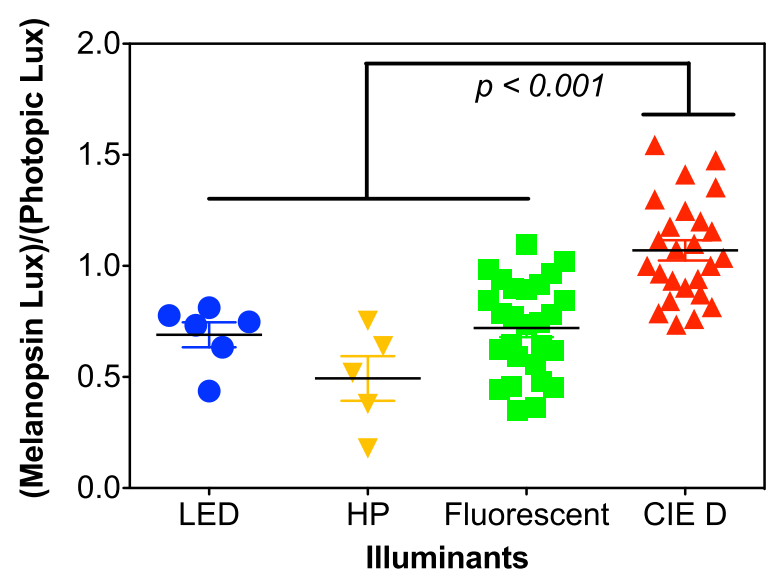

Fig. 4 The relative melanopsin activation with different illuminants at the same photopic illuminance levels 
potentially disrupt normal biological and physiological functions, causing various adverse health effects, such as circadian rhythm disruption, sleep disorders, mood disorders, and even cancer [20,21, 24-28]. For example, a latest study demonstrated that evening use of light-emitting-eReaders impaired sleep, circadian timing and next-morning alertness [49], although the real impact of evening use of eReaders on circadian rhythm will depend on prior light exposure history [50, 51]. Therefore, lighting (ambient and occupational lighting or display lighting) has become a public health issue [52]. To improve human quality of life and health, how to design artificial lights to optimize NIF functions (which are important for physical and mental health) as well as image-forming functions (which are important for normal daily function and life quality) has become an important issue. Additionally, primate ipRGCs responds excitatory to melanopsin activation, rod, L- and M-cone inputs but inhibitory to S-cone inputs [3]. This unique characteristic of its receptive field was shown to appear in pupillary recordings $[4,53]$. This chromatic opponency of ipRGCs may also be evolved to signal the large spectral changes, from bluish to orangish, produced at dawn and dusk to set the biological clock more precisely [3] and effectively [54]. Artificial lighting with unvaried chromaticities cannot trigger ipRGCs' responses as natural sunlight. However, more research is needed to fully understand how ipRGCs impact health and provide scientific guidelines for lighting design.

Finally, the discovery of ipRGCs will have great implication of light specification and regulation. Currently, regulations for lighting industry are based on photometry units (i.e. lux for illuminance or $\mathrm{cd} / \mathrm{m}^{2}$ for luminance). These units consider a particular visual function, the combination of L- and M-cone in the magnocellular pathway. Although many other visual functions could be considered, this function produces the additive photopic spectral-luminosity function $V_{\lambda}$, which is suitable for use in lighting industry [55]. However, melanopsin spectral sensitivity function is shifted to shorter wavelengths with respect to the overwhelmingly used $V_{\lambda}$. Therefore traditional photopic units cannot reflect the state of melanopsin activation that is important for health and perception. Recently, new approaches were proposed to cope with this issue by considering melanopsin activation $[56,57]$.

\section{Conclusions}

Human biology has evolved in direct relation and dependence with natural sunlight. Since the intrusion of massive artificial light sources, such as computer monitors, TV, self-illuminated personal electronic devices, indoor and street lighting, this relationship has been altered. Melanopsin activation in ipRGCs is important for many aspects of human functions, such as perception, cognition, circadian rhythm, sleep, mood and has great impact on health. Therefore, it is necessary for lighting and display designers to consider the new discovery of this century to improve, or at least affect as little as possible, human quality of life and health.

\section{Abbreviations}

CIE: Commission Internationale d'Eclairage; IF: image forming; IGL: intergeniculate leaflet; ipRGC: intrinsically photosensitive retinal ganglion cells; LED: Light Emission Diode; NIF: non-image forming; OPN: olivary pretectal nucleus; SCN: suprachiasmatic nucleus. 
Authors' contributions

DC conducted literature search, graphic preparation and data analysis and wrote the manuscript. PAB assisted in literature search, graphic preparation, data analysis and manuscript preparation. All authors read and approved the final manuscript.

\section{Authors' information}

DC is currently an Associate Professor in Department of Ophthalmology and Visual Sciences, University of Illinois at Chicago. He obtained his B.S. (Psychology) from Beijing University in 1991 and Ph.D. degree (Biopsychology with a concentration in Color Vision) from The University of Chicago in 2003. His research interests include visual perception (color perception, contrast sensitivity, temporal sensitivity, rod-cone interaction, impact of melanopsin activation on visual functions and pupil responses, ipRGC functions), circadian rhythm, retinal diseases and lighting. His Visual Perception Laboratory employs a combination of methods including psychophysics, electrophysiology, ocular imaging and genetics, aiming to understand the mechanisms that mediate our visual perception.

PAB is currently a researcher in the Institute of Research in Light, Environment and Vision from the National Scientific and Technical Research Council and National University of Tucuman - Argentina. He worked as a postdoctoral scholar in the Visual Perception Laboratory of the University of Illinois at Chicago with Dr. Cao. His work mainly concerns with rodcone interaction in the peripheral retina and the functional consequences of melanopsin activation in ipRGCs.

\section{Author details}

${ }^{1}$ Visual Perception Laboratory, Department of Ophthalmology and Visual Sciences, University of Illinois at Chicago, Chicago, IL 60612, USA. ${ }^{2}$ Institute of Research in Light, Environment and Vision, National University of Tucumán National Scientific and Technical Research Council, San Miguel de Tucumán, Tucumán 4000, Argentina.

\section{Received: 14 October 2015 Accepted: 17 December 2015}

\section{Published online: 24 December 2015}

\section{References}

1. Berson DM, Dunn FA, Takao M (2002) Phototransduction by retinal ganglion cells that set the circadian clock Science 295(5557):1070-1073

2. Hattar S, Liao HW, Takao M, Berson DM, Yau KW (2002) Melanopsin-containing retinal ganglion cells: architecture, projections, and intrinsic photosensitivity. Science 295:1065-1070

3. Dacey DM, Liao H, Peterson B, Robinson F, Smith VC, Pokorny J et al (2005) Melanopsin-expressing ganglion cells in primate retina signal color and irradiance and project to the LGN. Nature 433:749-754

4. Cao D, Nicandro N, Barrionuevo P (2015) A five-primary photostimulator suitable for studying intrinsically photosensitive retinal ganglion cell functions in humans. J Vision 15(1):27, 1-13. PMCID: 4528566

5. Barrionuevo P, Cao D (2014) Contributions of rhodopsin, cone opsins and melanopsin to postreceptoral pathways inferred from natural image statistics. J Opt Soc Am A 31(4):A131-A139, PMCID: 4117214

6. Barrionuevo P, Nicandro N, MCAnany JJ, Zele AJ, Gamlin PD, Cao D (2014) Assessing relative rod, cone and melanopsin contributions to pupil flicker responses. Invest Ophthalmol Vis Sci 55(2):719-727, PMCID: 3915766

7. Cao D, Barrionuevo P (2015) Estimating photoreceptor excitations from spectral outputs of a personal light exposure measurement device. Chronobiol Int 32(2):270-280

8. Do MTH, Yau K-W (2010) Intrinsically Photosensitive Retinal Ganglion Cells. Physiol Rev 90(4):1547-1581

9. Hattar S, Lucas RJ, Mrosovsky N, Thompson S, Douglas RH, Hankins MW et al (2003) Melanopsin and rod-cone photoreceptive systems account for all major accessory visual functions in mice. Nature 424:76-81

10. Lucas RJ, Hattar S, Takao M, Berson DM, Foster RG, Yau KW (2003) Diminished pupillary light reflex at high irradiances in melanopsin-knockout mice. Science 299:245-247

11. Thapan K, Arendt J, Skene DJ (2001) An action spectrum for melatonin suppression: evidence for a novel non-rod, non-cone photoreceptor system in humans. J Physiol 535(Pt 1):261-267

12. Brainard GC, Lewy AJ, Menaker M, Fredrickson RH, Miller LS, Weleber RG et al (1985) Effect of Light Wavelength on the Suppression of Nocturnal Plasma Melatonin in Normal Volunteersa. Ann NY Acad Sci 453(1):376-378

13. Altimus CM, Güler AD, Villa KL, McNeill DS, Legates TA, Hattar S (2008) Rods-cones and melanopsin detect light and dark to modulate sleep independent of image formation. Proc Natl Acad Sci U S A 105(50):19998-20003

14. Lupi D, Oster H, Thompson S, Foster RG (2008) The acute light-induction of sleep is mediated by OPN4-based photoreception. Nature Neurosci 11(9):1068-1073

15. Vandewalle G, Maquet P, Dijk D-J (2009) Light as a modulator of cognitive brain function. Trends Cogn Sci 13(10): 429-438

16. Lockley SW, Evans EE, Scheer FAJL, Brainard GC, Czeisler CA, Aeschbach D (2006) Short-wavelength sensitivity for the direct effects of light on alertness, vigilance, and the waking electroencephalogram in humans. Sleep-NY Westchester 29(2):161

17. Rahman SA, Flynn-Evans EE, Aeschbach D, Brainard GC, Czeisler CA, Lockley SW (2014) Diurnal spectral sensitivity of the acute alerting effects of light. Sleep 37(2):271-281

18. Badia P, Myers B, Boecker M, Culpepper J, Harsh J (1991) Bright light effects on body temperature, alertness, EEG and behavior. Physiol Behav 50(3):583-588

19. Cajochen C, Zeitzer JM, Czeisler CA, Dijk DJ (2000) Dose-response relationship for light intensity and ocular and electroencephalographic correlates of human alertness. Behav Brain Res 115:75-83

20. LeGates TA, Fernandez DC, Hattar S (2014) Light as a central modulator of circadian rhythms, sleep and affect. Nat Rev Neurosci 15(7):443-454

21. Golombek DA, Rosenstein RE (2010) Physiology of circadian entrainment. Physiol Rev 90(3):1063-1102

22. Rosenwasser AM (2009) Functional neuroanatomy of sleep and circadian rhythms. Brain Res Rev 61:281-306

23. Czeisler CA, Duffy JF, Shanahan TL, Brown EN, Mitchell JF, Rimmer DW et al (1999) Stability, precision, and near-24hour period of the human circadian pacemaker. Science 284:2177-2181 
24. Penev PD, Kolker DE, Zee PC, Turek FW (1998) Chronic circadian desynchronization decreases the survival of animals with cardiomyopathic heart disease. Am J Physiol 275:H2334-H2337

25. Barnard AR, Nolan PM (2008) When Clocks Go Bad: Neurobehavioural Consequences of Disrupted Circadian Timing. PLoS Genet 4(5), e1000040

26. McClung CA (2007) Circadian genes, rhythms and the biology of mood disorders. Pharmacol \& Therapeut 114(2): 222-232

27. Costa G, Haus E, Stevens R (2010) Shift work and cancer - considerations on rationale, mechanisms, and epidemiology. Scand J Work Environ Health 36(2):163-179

28. Blask D, Brainard G, Gibbons R, Lockley S, Stevens R, Motta M. Council on Science and Public Health Report 4. Light Pollution: Adverse Health Effects of Nighttime Lighting. American Medical Association House of Delegates Annual Meeting, 2012 June. Report No.

29. Feigl B, Zele AJ (2014) Melanopsin-Expressing Intrinsically Photosensitive Retinal Ganglion Cells in Retinal Disease. Optom Vis Sci 9(18):894-903

30. Pokorny J, Smith VC (1997) How much light reaches the retina? In C.R. Cavonius (ed), Colour Vision Deficiencies XIII. Doc Ophthalmol Proc Ser 59:491-511

31. Gamlin PD, McDougal DH, Pokorny J, Smith VC, Yau KW, Dacey DM (2007) Human and macaque pupil responses driven by melanopsin-containing retinal ganglion cells. Vision Res 47:946-954

32. McDougal DH, Gamlin PD (2010) The influence of intrinsically-photosensitive retinal ganglion cells on the spectral sensitivity and response dynamics of the human pupillary light reflex. Vision Res 50(1):72-87

33. Joyce DS, Feigl B, Cao D, Zele AJ (2015) Temporal characteristics of melanopsin inputs to the human pupil light reflex. Vision Res 107:58-66

34. Park JC, MCAnany JJ (2015) Effect of stimulus size and luminance on the rod-, cone-, and melanopsin-mediated pupillary light reflex. J Vision 15:13, 1

35. Berson DM (2003) Strange vision: ganglion cells as circadian photoreceptors. Trends Neurosci 26(6):314-320

36. Allen AE, Storchi R, Martial FP, Petersen RS, Montemurro MA, Brown TM et al (2014) Melanopsin-driven light adaptation in mouse vision. Curr Biol 24(21):2481-2490

37. Barnard AR, Hattar S, Hankins MW, Lucas RJ (2006) Melanopsin regulates visual processing in the mouse retina Curr Biol 16(4):389-395

38. Hankins MW, Lucas RJ (2002) The primary visual pathway in humans is regulated according to long-term light exposure through the action of a nonclassical photopigment. Curr Biol 12:191-198

39. Zaidi FH, Hull JT, Peirson SN, Wulff K, Aeschbach D, Gooley JJ et al (2007) Short-wavelength light sensitivity of circadian, pupillary, and visual awareness in humans lacking an outer retina. Curr Biol 17(24):2122-2128

40. Brown TM, Tsujimura S, Allen AE, Wynne J, Bedford R, Vickery G et al (2012) Melanopsin-based brightness discrimination in mice and humans. Curr Biol 22(12):1134-1141

41. Horiguchi H, Winawer J, Dougherty RF, Wandell BA (2013) Human trichromacy revisited. Proc Natl Acad Sci U S A 110(3):E260-E269

42. Cao D, Barrionuevo PA (2015) Melanopsin-mediated color percepts. International Colour Vision Society Symposium, Sendai, Japan, Abstract

43. Spitschan M, Datta R, Stern AM, Brainard DH, Aguirre GD. Human visual cortex responses to rapid cone and melanopsin directed flicker. J Neurosci. 2015: In press.

44. Tsujimura S, Hamazono N, Okajima K (2014) Temporal contrast sensitivity function based on cones and melanopsin photoreceptors. J Vision 14(10):593

45. Lee BB (2011) Visual pathways and psychophysical channels in the primate. J Physiol 589:41-47

46. Dacey DM (2000) Parallel pathways for spectral coding in primate retina. Annu Rev Neurosci 23:743-775

47. Ruderman DL, Cronin TW, Chiao CC (1998) Statistics of cone responses to natural images: Implications for visual coding. J Opt Soc Am A 15(8):2036-2045

48. Jacobs GH, Nathans J (2009) The evolution of primate color vision. Sci Am 300(4):56-63

49. Chang AM, Aeschbach D, Duffy JF, Czeisler CA (2015) Evening use of light-emitting eReaders negatively affects sleep, circadian timing, and next-morning alertness. Proc Natl Acad Sci U S A 112:1232-1237

50. Chang AM, Scheer FAJL, Czeisler CA (2011) The human circadian system adapts to prior photic history. J Physiol 589(5):1095-1102

51. Zeitzer JM, Friedman L, Yesavage JA (2011) Effectiveness of evening phototherapy for insomnia is reduced by bright daytime light exposure. Sleep Med 12(8):805-807

52. Pauley SM (2004) Lighting for the human circadian clock: recent research indicates that lighting has become a public health issue. Med Hypotheses 63(4):588-596

53. Spitschan M, Jain S, Brainard DH, Aguirre GK (2014) Opponent melanopsin and S-cone signals in the human pupillary light response. Proc Natl Acad Sci U S A 111(43):15568-15572

54. Walmsley L, Hanna L, Mouland J, Martial FP, West A, Smedley AR, et al. Colour as a signal for entraining the mammalian circadian clock. PLoS Biol. 2015; DOl: 10.1371/journal.pbio.1002127:1-20.

55. Lennie P, Pokorny J, Smith VC (1993) Luminance. J Opt Soc Am A 10(6):1283-1293

56. Lucas RJ, Peirson SN, Berson DM, Brown TM, Cooper HM, Czeisler CA et al (2014) Measuring and using light in the melanopsin age. Trends Neurosci 37(1):1-9

57. Rea MS (2015) The lumen seen in a new light: Making distinctions between light, lighting and neuroscience. Lighting Res Technol 47(3):259-280 